\title{
Micellisation and aggregation of self-propelled hard circulangles.
}

\author{
Timofei Kruglov ${ }^{* 1}$ and Alexander Borisov ${ }^{1,2}$ \\ ${ }^{1}$ Research and Technology Office Russia, Robert Bosch OOO \\ ul. Marshala Govorova 49 A, 198095 St-Petersburg, Russia \\ Email: timofey.kruglov@bosch.com, Web page: https://www.bosch.com/research/ \\ ${ }^{2}$ Robert Bosch GmbH, Robert-Bosch-Campus 1, 71272 Renningen, Germany
}

\begin{abstract}
Self-organization in active materials, inspired by biological systems, shows many features, not found in passive materials [2]. Self-propelled Brownian spheres undergo phase separation [3], rodlike particles show unusual defects behavior, which was confirmed experimentally [1]. Both types of particles, however, have a center of symmetry.

In order to explore the influence of shape asymmetry, a new 2D shape, named a circulangle, is introduced. It is composed of a circle, wedged into an angle, such that the angle's sides attach to the circle tangentially thus preserving continuous curvature. It is characterized by an elongation factor $L / R$, which is 1 for a circle (Fig. 1a). Monte Carlo simulations of two-dimensional hard Brownian circulangles are presented. Self-propellence is introduced by the bias of MC moves along particles' axes. Upon such MC sampling the system undergoes transition from homogeneous isotropic state (Fig. 1b ) to micellar structures (Fig. 1d) arranged in clusters/networks (Fig. 1c).

Circulangles appear to be the first known self-propelled particles, which form micelles without attractive forces.

The newly discovered phenomenon may have wide implications in design of (meta)materials for energy conversion and storage, sensorics, micromechanics and targeted drug delivery.
\end{abstract}

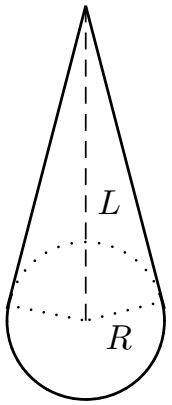

(a) Single circulangle particle

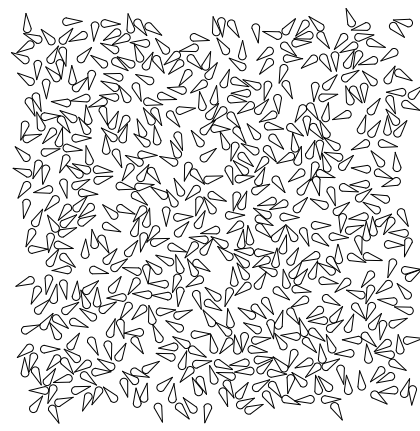

(b) Isotropic, homogeneous

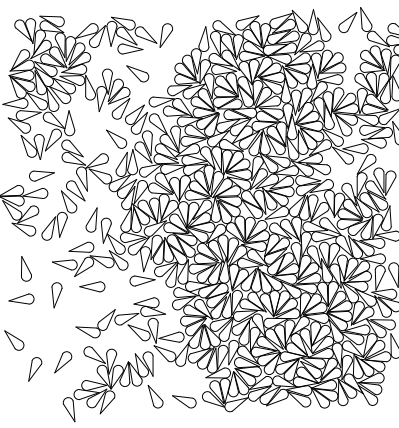

(c) Micellar

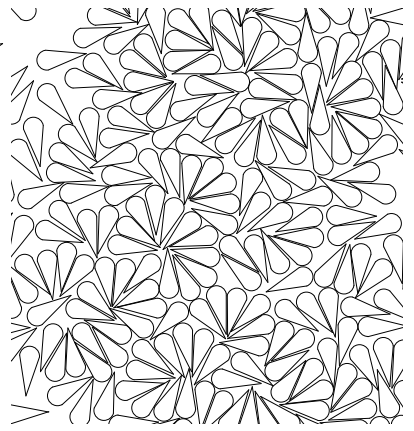

(d) Micelles zoomed

Figure 1: Circulangles with $L / R=4$

\section{REREFERNCES}

[1] Stephen J DeCamp et al. "Orientational order of motile defects in active nematics". In: Nature materials 14.11 (2015), pp. 1110-1115.

[2] Michael F Hagan and Aparna Baskaran. "Emergent self-organization in active materials". In: Current Opinion in Cell Biology 38 (2016), pp. 74-80.

[3] Gabriel S. Redner, Michael F. Hagan, and Aparna Baskaran. "Structure and Dynamics of a Phase-Separating Active Colloidal Fluid". In: Phys. Rev. Lett. 110 (5 2013), p. 055701. 